\section{The Effect of Anti-Nuclear An- tibody Positivity on Immune Thrombocytopenic Purpura and its Clinical Course}

\author{
Loui A Ezzat ${ }^{1 *}$ and Hatoon M Ezzat ${ }^{2}$ \\ ${ }^{1}$ College of Medicine, King Saud University, Riyadh, Saudi Arabia \\ ${ }^{2}$ Department of Hematology, University of British Columbia, Vancouver, \\ Canada
}

\begin{abstract}
\section{Objective}

Immune Thrombocytopenic Purpura (ITP) is an autoimmune-mediated disease. Patients with primary ITP are occasionally found to have an Antinuclear Antibody (ANA) titer $\geq 1: 160$. Our objective was to study the effect of ANA on ITP and its clinical course.

\section{Methods}

This was a retrospective chart review of 765 adult patients with ITP, followed regularly at St. Paul's Hospital, Vancouver, Canada; 120 patients who had an ITP diagnosis and had ANA testing during their initial work-up were included. An ANA titer $\geq 1: 160$ was correlated with clinical presentation including steroid responsiveness, platelet count at presentation, and autoimmune disease development. This study took place in St. Paul's Hospital, Vancouver, British Columbia, Canada between the months of June 2014, till January 2015. Results

Of 24 patients with ANA titers $\geq 1: 160,10(58.8 \%)$ were steroid-resistant, compared to $9(29.0 \%)$ of 31 patients with ANA titers $<1: 160(P=0.044)$. Five $(20.8 \%)$ patients with ANA titers $\geq 1: 160$ developed other autoimmune diseases after diagnosis with ITP, compared to only one (1\%) patient with an ANA titer $<1: 160(\mathrm{P}=0.001)$. Patients with ANA titers $\geq 1: 160$ had a lower platelet count than patients with ANA titers $<1: 160,(P<0.001)$.

\section{Conclusion}

We analyzed the relationship between high ANA and ITP clinical course. Compared to patients with ANA titers $<1: 160$, those with ANA titers $\geq 1: 160$ were steroid-resistant, had a higher risk of developing other autoimmune diseases, and presented with a lower platelet count.

\section{Keywords: ANA; ITP; SLE; Steroids}

*Corresponding author: Loui A Ezzat, Department of Medicine, King Saud University, Riyadh, Saudi Arabia Tel: +966 551989989, E-mail: Loui.ezzat@yahoo.com

Citation: Ezzat LA, Ezzat HM (2017) The Effect of Anti-Nuclear Antibody Positivity on Immune Thrombocytopenic Purpura and its Clinical Course. J Hematol Blood Transfus Disord 4: 015.

Received: January 23, 2017; Accepted: March 14, 2017; Published: March 30,2017

\section{Introduction}

Immune Thrombocytopenic Purpura (ITP) is defined as an autoimmune disease characterized by isolated thrombocytopenia with a platelet count $\leq 100 \times 109 / \mathrm{L}$ resulting from accelerated destruction of antibody-coated platelets by tissue macrophages, predominantly in the spleen. ITP can be primary with no identifiable or specific precipitating factor, while secondary ITP is associated with an underlying disease [1]. ITP is classified into three phases based on disease duration: newly diagnosed, less than 3 months; persistent, between 3 and 12 months; and chronic, more than 12 months [1]. While the majority of affected children have secondary short-lived ITP, adults usually have primary chronic ITP [2].

In primary ITP, it is believed that the patient's B-cells produce IgG antibodies against platelet membrane glycoproteins, resulting in antibody-mediated destruction [3]. A subset of patients with ITP are positive for Antinuclear Antibodies (ANA) and antiphospholipid antibodies, in addition to antiplatelet antibodies. However, this does not qualify them as having secondary ITP if they do not meet the criteria for Systemic Lupus Erythematosus (SLE) and antiphospholipid syndrome $[4,5]$. The efficacy of regular testing for ANA in patients presenting with a low platelet count remain controversial in children and young adults [6].

Additionally, ANA-positive patients have been reported to have an unusual response to treatment [7]. A pilot study by Abbasi et al., [7] showed that adult patients with ITP who are ANA-positive have a poor response to steroid therapy; close monitoring was suggested for these patients [7]. Furthermore, Khellaf et al., [8] suggested the use of hydroxychloroquine as a second-line drug in patients with SLE-associated ITP as well as ANA-positive ITP. This previous study showed that $50 \%$ of patients with ANA-positive ITP without definite SLE showed a good response and tolerated hydroxychloroquine well [8]. Moreover, Hazzan et al., [9] studied the risk of developing SLE in ITP patients and found that all patients who developed SLE were women and ANA-positive; however, 64\% of ANA-positive ITP patients did not develop SLE [9].

In this single-center chart review, we reviewed and analyzed the data of adult patients with ITP, focusing on ANA positivity and its effect on treatment and future prognosis. To determine the relationship of ANA positivity on treatment outcome and future prognosis, we retrospectively reviewed the data of adult patients with ITP. We hypothesized that patients with a higher degree of ANA positivity would have worse outcomes and prognosis.

\section{Materials and Methods}

This is a retrospective chart review of patients at St.Paul's Hospital, Vancouver, British Columbia, Canada between the months of June 2014, till January 2015. We conducted a retrospective chart review of 765 adult patients with ITP, who were regularly followed up at St. Paul's Hospital, Vancouver, British Columbia, Canada. Patients included were all diagnosed with primary ITP, aged $\geq 15$ years, and who had their ANA titers tested at the time of diagnosis. Exclusion criteria 
included diagnosis of hepatitis $\mathrm{C}$, hepatitis B, or human immunodeficiency virus, as well as diagnosis of autoimmune diseases prior to the diagnosis of ITP.

Data extracted included date of birth, age at time of diagnosis, gender, ANA titer, follow-up period, treatment used (if any), response to treatment, platelet level before and after treatment, diagnosis of other autoimmune disease, signs and symptoms of autoimmune disorders at the time of diagnosis of ITP, direct family history of autoimmune diseases, complete blood count with differential, C-reactive protein, and erythrocyte sedimentation rate.

Diagnosis of ITP was made by a hematologist, and patients who subsequently developed other autoimmune diseases were diagnosed strictly by rheumatologists. ANA titer was considered positive if the ANA titer was $\geq 1: 160$, as many centers consider ANA titer $1: 80$ as equivocal. Treatment was based on the treating hematologist's preference; however, steroids were the first line of therapy. Patients were considered steroid-resistant only if a steroid trial of at least 2 weeks was attempted and the patient did not improve. Other patients were considered steroid-dependent when it was not possible to taper the steroid dose without a drop in platelet count. Other treatment methods included intravenous immunoglobulin, azathioprine, rituximab, and romiplostim; moreover, splenectomy was performed in some patients.

Data were analyzed using a chi-square test, Fisher's exact test, or Wilcoxon rank-sum test as appropriate. $\mathrm{P}$ value $<0.05$ was considered statistically significant.

This analysis was performed in accordance with requirements of the Institutional Research Ethics Boards at St. Paul's Hospital, and ethical approval obtained.

\section{Results}

In total, 765 charts were reviewed. One hundred and twenty patients were included based on criteria and availability of ANA testing. Out of 120 patients, 24 patients were ANA positive. ANA titers for these patients are summarized in table 1.

\begin{tabular}{|c|c|c|c|}
\hline ANA Titer & All Patients $(\mathbf{n = 1 2 0})$ & ANA $<1: \mathbf{1 6 0}(\mathbf{n}=\mathbf{9 6})$ & ANA $\geq 1: \mathbf{1 6 0}(\mathbf{n}=\mathbf{2 4})$ \\
\hline 0 & $76(63.3)$ & $76(79.2)$ & $0(0.0)$ \\
\hline $1: 40$ & $10(8.3)$ & $10(10.4)$ & $0(0.0)$ \\
\hline $1: 80$ & $10(8.3)$ & $10(10.4)$ & $0(0.0)$ \\
\hline $1: 160$ & $11(9.2)$ & $0(0.0)$ & $11(45.8)$ \\
\hline $1: 320$ & $4(3.3)$ & $0(0.0)$ & $4(16.7)$ \\
\hline $1: 640$ & $6(5.0)$ & $0(0.0)$ & $6(25.0)$ \\
\hline $1: 1280$ & $3(2.5)$ & $0(0.0)$ & $3(12.5)$ \\
\hline
\end{tabular}

Table 1: Antinuclear Antibody (ANA) titers in patients with immune thrombocytopenic purpura.

*ANA, antinuclear antibody

The majority $(n=80 ; 66.7 \%)$ of patients diagnosed with ITP were women. While $20(25 \%)$ female patients with ITP were found to have ANA titers $\geq 1: 160$, only $4(10 \%)$ male patients were found to have ANA titers $\geq 1: 160$. Moreover, a higher percentage (83.3\%) of patients with ANA titers $\geq 1: 160$ were women compared with that among patients with ANA titers $<1: 160(62.5 \%)$. The mean age at the time of diagnosis of ITP was 41.0 years (standard deviation [SD] 17.6). Moreover, the mean age at the time of diagnosis was non-significantly lower in patients with ANA titers $\geq 1: 160(37.8$ years, SD 19.2) than that in those with ANA titers $<1: 160$ (41.8 years, SD 17.2).

Patients included in this study were treated according to their symptoms, including $\mathrm{C}$, prolonged epistaxis, overt hematuria, and gingival bleeding associated with a platelet count of $\leq 30,000 / \mathrm{mL}$.

While the majority of patients $(n=69,57.5 \%)$ did not need any intervention, the remainder of patients required intervention to control their symptoms. Steroids were adequate to control the symptoms of most patients needing intervention $(n=32,26.7 \%)$. However, in a number of patients ( $\mathrm{n}=19,15.8 \%$ of all ITP patients), steroids failed to control these symptoms. When comparing all ANA-positive patients with ANA-negative patients in our study, we found that significantly more patients ( $\mathrm{n}=10,58.8 \%)$ with ANA titers $\geq 1: 160$ were steroid-resistant than those with ANA titers $<1: 160(\mathrm{n}=9,29.0 \%$; $\mathrm{P}=0.044)$. Patients who've failed steroid thearapy have been treated with various modalities. The different treatment modalities used are summarized in table 2 . The response to steroid therapy is summarized in table 3.

\begin{tabular}{|c|c|c|c|}
\hline $\begin{array}{l}\text { Treatment } \\
\text { Used }\end{array}$ & $\begin{array}{l}\text { All Patients } \\
(n=120)\end{array}$ & ANA $<1: 160(n=96)$ & $\begin{array}{c}\text { ANA } \\
\geq 1: 160(n=24)\end{array}$ \\
\hline None & $69(57.5)$ & $64(66.7)$ & $5(20.8)$ \\
\hline IVIG & $3(2.5)$ & $1(1.0)$ & $2(8.3)$ \\
\hline Rituximab & $2(1.7)$ & $1(1.0)$ & $1(4.2)$ \\
\hline Steroids & $32(26.7)$ & $22(22.9)$ & $10(41.7)$ \\
\hline Azathioprine & $2(1.7)$ & $0(0.0)$ & $2(8.3)$ \\
\hline Romiplostim & $1(0.8)$ & $0(0.0)$ & $1(4.2)$ \\
\hline Splenectomy & $11(9.2)$ & $8(8.3)$ & $3(12.5)$ \\
\hline
\end{tabular}

Table 2: Treatments for patients with immune thrombocytopenic purpura. *ANA, antinuclear antibody

\begin{tabular}{|c|c|c|c|c|}
\hline Variable & All & $\begin{array}{c}\text { ANA } \\
<1: 160(n=96)\end{array}$ & $\begin{array}{c}\text { ANA } \\
\geq 1: 160(n=24)\end{array}$ & P Value \\
\hline \multicolumn{4}{|l|}{ Steroid resistance } & \multirow{3}{*}{0.044} \\
\hline Responsive & $29(60.4)$ & $22(71.0)$ & $7(41.2)$ & \\
\hline Resistant/dependent & 19(39.6) & $9(29.0)$ & $10(58.8)$ & \\
\hline \multicolumn{4}{|c|}{ Recent diagnosis of other autoimmune disorders } & \multirow{3}{*}{0.001} \\
\hline No & $114(95.0)$ & $95(99.0)$ & 19(79.2) & \\
\hline Yes & $6(5.0)$ & $1(1.0)$ & $5(20.8)$ & \\
\hline \multicolumn{4}{|c|}{ Platelet level before treatment } & \multirow{5}{*}{$<0.001$} \\
\hline Missing, $n(\%)$ & $2(1.7)$ & $1(1.0)$ & $1(4.2)$ & \\
\hline Median (IQR) & $\begin{array}{c}57.000 / \mathrm{mL} \\
(17.000 / \mathrm{mL}, \\
94.000 / \mathrm{mL})\end{array}$ & $\begin{array}{c}65.000 / \mathrm{mL} \\
(30.000 / \mathrm{mL} \\
103.000 / \mathrm{mL})\end{array}$ & $\begin{array}{c}16.000 / \mathrm{mL} \\
(13.000 / \mathrm{mL}, \\
49.000 / \mathrm{mL})\end{array}$ & \\
\hline Mean (SD) & $\begin{array}{c}59.900 / \mathrm{mL} \\
(41.900 / \mathrm{mL})\end{array}$ & $\begin{array}{c}66.300 / \mathrm{mL} \\
(41.500 / \mathrm{mL})\end{array}$ & $\begin{array}{c}33.200 / \mathrm{mL} \\
(32.700 / \mathrm{mL})\end{array}$ & \\
\hline Range & $\begin{array}{c}(0.000 / \mathrm{mL} \\
146.000 / \mathrm{mL})\end{array}$ & $\begin{array}{c}(0.000 / \mathrm{mL} \\
146.000 / \mathrm{mL})\end{array}$ & $\begin{array}{c}(2.000 / \mathrm{mL} \\
126.000 / \mathrm{mL})\end{array}$ & \\
\hline
\end{tabular}

Table 3: Clinical characteristics based on high or low Antinuclear Antibody (ANA) titers.

All values are $n(\%)$, except as otherwise indicated. IQR, Interquartile Range; $\mathrm{SD}$, Standard Deviation

Only one patient had a direct family history of SLE; this patient had an ANA titer of 1:80. However, 5(20.8\%) patients with ANA titers $\geq 1: 160$ had developed further autoimmune diseases after being diagnosed with ITP, compared to only one (1\%) patient with an ANA titer $<1: 160(\mathrm{P}=0.001)$; these diseases included systemic lupus erythematosus and anti-phospholipid syndrome. Moreover, patients with ANA 
titers $\geq 1: 160$ had a significantly lower platelet count (mean, 33.000/ $\mathrm{mL}$ ) than patients with ANA titers $<1: 160$ (mean, 59.9; $\mathrm{P}<0.001$ ). Table 3 summarizes these clinical characteristics.

\section{Discussion}

This study explored the relationship between a positive ANA titer $(\geq 1: 160)$ and ITP clinical course and outcome. Three main aspects of patients with a positive ANA titer were statistically compared to that of patients with an equivocal or negative ANA titer: steroid resistance, progression to other autoimmune disease, and platelet level at presentation.

It is well accepted that steroids are the first-line treatment modality in ITP. Several studies that evaluated a total of 442 patients with ITP found that $81 \%$ of hematologists used steroids as a first-line treatment [10-14].

Patients with an ANA titer $\geq 1: 160$ were more likely to have steroid resistance than patients with an ANA titer $<1: 160$. The 2008 study of Abbasi et al., [7] also supports this finding, as the platelet count remained significantly lower after treatment in ANA-positive patients. Additionally, Khellaf et al [8] suggested hydroxychloroquine as a second-line therapy in patients with an ANA titer $\geq 1: 160$, as the patients included in their study were all steroid-resistant. In contrast to our findings, a retrospective chart review of 1791 ITP case showed no difference in response to steroids between ANA-negative and ANA-positive ITP patients; nevertheless, ANA-positive patients had a shorter remission period post-splenectomy [15].

Moreover, a higher percentage of ITP patients with ANA titers $\geq 1: 160$ showed progression to other autoimmune diseases compared to the percentage in those with ANA titers $<1: 160$. This agrees with the findings of Hazzan et al., [9] who studied the risk of progression to SLE in ITP patients with positive ANA titers. He found that all patients who showed progression to SLE had ANA titers $\geq 1: 160$, despite the fact that the majority of ITP patients with ANA titers $\geq 1: 160$ did not develop SLE. In contrast, Altintas et al. studied 36 adults with ITP and positive ANA titers, and only one of them developed Sjogren syndrome, leading them to the belief that ANA positivity alone is not a good predictor for the risk of developing further autoimmune diseases [16].

Finally, ITP patients with ANA titers $\geq 1: 160$ presented with a much lower platelet count than did patients with ANA titers $<1: 160$. We were unable to find any previous reports comparing platelet count at presentation according to the ANA titer.

Thus, our study findings indicate that ANA titers $\geq 1: 160$ is a poor prognostic factor for ITP outcomes, as it is associated with steroid resistance, progression to other autoimmune disease, and a lower platelet count at presentation.

We believe that our study could have been more valuable if it would have been a prospective study, as it may shed the light on aspects we could not have anticipated. Moreover, we believe our biggest disadvantage was that the majority of ITP patients did not have their ANA levels measured which led to a relatively small sample size.

\section{Conclusion}

ITP is a disease with a variable presentation; however, ANA titer levels could be a useful predictor for prognosis. Patients with ITP and
ANA titers $\geq 1: 160$ were prone to exhibit steroid resistance, have a higher risk of developing other autoimmune diseases, and to present with a lower platelet count than patients with ANA titers $<1: 160$.

\section{References}

1. Rodeghiero F, Stasi R, Gernsheimer T, Michel M, Provan D, Arnold DM, et al. (2009) Standardization of terminology, definitions and outcome criteria in immune thrombocytopenic purpura of adults and children: report from an international working group. Blood 113: 2386-2393.

2. Stasi R, Stipa E, Masi M, Cecconi M, Scimò MT, et al. (1995) Long-term observation of 208 adults with chronic idiopathic thrombocytopenic purpura. Am J Med 98: 436-442.

3. Chong BH (2009) Primary immune thrombocytopenia: understanding pathogenesis is the key to better treatments. J Thromb Haemost 7: 319-321.

4. Hochberg MC (1997) Updating the American College of Rheumatology revised criteria for the classification of systemic lupus erythematosus. Arthritis Rheum 40: 1725.

5. Miyakis S, Lockshin MD, Atsumi T, Branch DW, Brey RL, et al. (2006) International consensus statement on an update of the classification criteria for definite antiphospholipid syndrome (APS). J Thromb Haemost 4: 295-306.

6. Neunert C, Crowther M, Crowther M, Cohen A, Solberg L Jr, et al. (2011) The American Society of Hematology 2011 evidence-based practice guideline for immune thrombocytopenia. Blood 117: 4190-4207.

7. Abbasi SY, Milhem M, Zaru L (2008) A positive antinuclear antibody test predicts for a poor response to initial steroid therapy in adults with idiopathic thrombocytopenic purpura. Ann Hematol 87: 459-462.

8. Khellaf M, Chabrol A, Mahevas M, Roudot-Thoraval F, Limal N, et al. (2014) Hydroxychloroquine is a good second-line treatment for adults with immune thrombocytopenia and positive antinuclear antibodies. Am J Hematol 89: 194-198.

9. Hazzan R, Mukamel M, Yacobovich J, Yaniv I, Tamary H, et al. (2006) Risk factors for future development of systemic lupus erythematosus in children with idiopathic thrombocytopenic purpura. Pediatr Blood Cancer 47: 657-659.

10. Yang R, Han ZC (2000) Pathogenesis and management of chronic idiopathic thrombocytopenic purpura: an update. Int J Hematol 71: 18-24.

11. Lu KH, George JN, Vesely SK, Terrell DR (2014) Management of primary immune thrombocytopenia, 2012: a survey of oklahoma hematologists-oncologists. Am J Med Sci 347: 190-194.

12. Weide R, Feiten S, Friesenhahn V, Heymanns J, Kleboth K, et al. (2016) Outpatient management of patients with Immune Thrombocytopenia (ITP) by hematologists 1995-2014. Oncol Res Treat 39: 41-44.

13. Provan D, Stasi R, Newland AC, Blanchette VS, Bolton-Maggs $P$, et al. (2010) International consensus report on the investigation and management of primary immune thrombocytopenia. Blood 115: 168-186.

14. Kitchens CS (1977) Amelioration of endothelial abnormalities by prednisone in experimental thrombocytopenia in the rabbit. J Clin Invest 60: 1129-1134.

15. Li HQ, Zhang L, Zhao H, Ji LX, Yang RC, et al. (2005) Chronic idiopathic thrombocytopenic purpura in adult Chinese patients: a retrospective single-centered analysis of 1791 cases. Chin Med J 118: 34-37.

16. Altintas A, Ozel A, Okur N, Okur N, Cil T, et al. (2007) Prevalence and clinical significance of elevated antinuclear antibody test in children and adult patients with idiopathic thrombocytopenic purpura. J Thromb Thrombolysis 24: 163-168. 